\title{
Komponisten-Gesamtausgaben im digitalen Zeitalter: Perspektiven und Reflexionen am Beispiel Ludwig van Beethovens
}

\author{
Christine Siegert, Bonn
}

DOI: $10.25366 / 2020.95$

Zitation: Christine Siegert, "Komponisten-Gesamtausgaben im digitalen Zeitalter: Perspektiven und Reflexionen am Beispiel Ludwig van Beethovens", in: Brückenschläge zwischen Musikwissenschaft und Informatik. Theoretische und praktische Aspekte der Kooperation, in Verbindung mit der Fachgruppe Digitale Musikwissenschaft hrsg. von Stefanie Acquavella-Rauch, Andreas Münzmay und Joachim Veit (= Musikwissenschaft: Aktuelle Perspektiven. Bericht über die Jahrestagung der Gesellschaft für Musikforschung 2019 in Paderborn und Detmold, Bd. 3), Detmold, Musikwissenschaftliches Seminar der Universität Paderborn und der Hochschule für Musik Detmold, 2020, S. 61-76, DOI: $10.25366 / 2020.95$ 


\title{
Komponisten-Gesamtausgaben im digitalen Zeitalter: Perspektiven und Reflexionen am Beispiel Ludwig van Beethovens
}

\author{
CHRISTINE SIEGERT, BONN
}

Der Bereich der Editionsphilologie kann derzeit als einer der lebendigsten und innovativsten in der Musikwissenschaft gelten. In verschiedenen Editionsprojekten wurden und werden digitale Editionsanteile verschiedenster Art erprobt. Die Spannbreite reicht vom digitalen Kritischen Bericht und der digitalen Librettoedition bei OPERA - Spektrum des europäischen Musiktheaters in Einzeleditionen ${ }^{1}$ über die exemplarische digitale Freischütz-Edition ${ }^{2}$ bis hin zu digitalen Dokumenteneditionen, die die gedruckten Bände begleiten, wie bei der Max-Reger- ${ }^{3}$ und RichardStrauss-Ausgabe. ${ }^{4}$ Das Projekt Beethovens Werkstatt, ${ }^{5}$ das die genetische Textkritik mit digitalen Darstellungsmöglichkeiten auf innovative Weise verbindet, hat die Edition eines kompletten Werks allerdings mit Bedacht ans Ende des Arbeitsprogramms gestellt. ${ }^{6}$ Sie soll den krönenden Abschluss des Projekts bilden. Im Sommer 2019 ist die Digitale Mozart-Edition online gegangen, ${ }^{7}$ die nach der lediglich digitalisierten Neuen Mozart-Ausgabe (NMA) nun eine digitale Edition auf MEI-Basis realisiert, bei der allerdings als "Referenztext" mit der NMA eine traditionelle Druckausgabe die Grundlage der Edition bleibt.

Insofern steht eine genuin digitale Edition noch aus, denn diese müsste ausgehend von den Möglichkeiten des Mediums Internet her konzipiert werden. Zwei Aspekte scheinen mir dabei von zentraler Bedeutung zu sein: Pluralität und Vernetzung. Anhand von vier Beispielen aus dem Schaffen Ludwig van Beethovens sollen im Folgenden die Grenzen traditioneller Druckausgaben anhand der Neuen Beethoven-Gesamtausgabe (NGA) und das Potential einer Digitalen Beethoven-Edition aufgezeigt und damit erste skizzenhafte Überlegungen zu einer solchen Edition zur Diskussion gestellt werden.

<http://www.opera.adwmainz.de/informationen.html> (09.05.2020).

<https://freischuetz-digital.de/> (09.05.2020).

<https://www.max-reger-institut.de/de/reger-werkausgabe> (23.08.2020).

<https://www.richard-strauss-ausgabe.de/> (23.08.2020).

5 Das Projekt ist am Musikwissenschaftlichen Seminar Detmold/Paderborn und dem Beethoven-Haus Bonn angesiedelt und wird von Bernhard R. Appel und Joachim Veit geleitet.

6 <https://beethovens-werkstatt.de/projekt/> (09.05.2020). Als Modul 5 sind „Drei Modelleditionen der DiabelliVariationen op. $120 "$ geplant.

7 <https://dme.mozarteum.at/musik/edition/> (23.08.2020). Für den Hinweis in der Diskussion danke ich Norbert Dubowy. 


\section{Die Grenzen der analogen Edition}

a) Steiners Ausgaben von op. 91-93

Als der Wiener Verleger Sigmund Anton Steiner ab 1816 die Originalausgaben von Beethovens Schlachtensymphonie Wellingtons Sieg oder die Schlacht bei Vittoria op. 91 sowie der Siebten und Achten Symphonie op. 92 bzw. 93 publizierte, beschränkte er sich nicht auf die Ausgaben in Stimmen und in Partitur, sondern brachte zusätzlich Ausgaben für Bläserensemble, Streichquintett, Klaviertrio, Klavier vier- und zweihändig heraus, um „alle Freunde der Tonkunst in dem [recte: den] Genusse dieser herrlichen Kunstwerke [...] zu sezten". Steiner unterscheidet nicht zwischen "Original" und "Bearbeitung", sondern führt die Ausgaben unterschiedslos in absteigender Besetzung an. Alle Ausgaben würden "an ein und demselben Tag aus[ge]geben" und „unter der unmittelbaren Revision ihres Schöpfers Herrn Ludwig van Beethoven, vollendet." Beethoven war in unterschiedlichem Maße an den Ausgaben beteiligt. Den zweihändigen Klavierauszug zu Wellingtons Sieg stellte er selbst her, ${ }^{9}$ von der Siebten Symphonie existiert ein entsprechendes Fragment. ${ }^{10}$ Auch darüber hinaus ist davon auszugehen, dass Beethoven mit der Publikationsstrategie des Verlegers einverstanden war: Am 1. Februar 1815 bat er Steiner, die Auszüge selbst herzustellen, "doch sollen alle von mir übersehen, und wo es nöthig, verbessert werden". ${ }^{11}$

Steiners mit Beethoven abgestimmtes Publikationskonzept legt es nahe, die Werkkomplexe mit den multiplen Werkausprägungen jeweils als Einheit zu betrachten. Als gedruckte Ausgabe muss sich die Neue Beethoven-Gesamtausgabe allerdings auf die Orchesterpartituren sowie die Klavierauszüge beschränken. ${ }^{12}$

8 Zitiert nach der Anzeige für die beiden Symphonien im Klavierauszug von Wellingtons Sieg (Exemplar: Beethoven-Haus Bonn, Sammlung H.C. Bodmer, HCB C op. 91).

9 Vgl. Ludwig van Beethoven. Thematisch-bibliographisches Werkverzeichnis, bearbeitet von Kurt Dorfmüller, Norbert Gertsch und Julia Ronge unter Mitarbeit von Gertraut Haberkamp und dem Beethoven-Haus Bonn, revidierte und wesentlich erweiterte Neuausgabe des Verzeichnisses von Georg Kinsky und Hans Halm, München 2014, Bd. 1, S. 575.

10 Vgl. ebd., S. 591.

11 Ludwig van Beethoven. Briefwechsel Gesamtausgabe, hrsg. von Sieghard Brandenburg, Bd. 3: 1814-1816, München 1996, S. 109, Nr. 780.

12 Ludwig van Beethoven, Ouverturen und Wellingtons Sieg, hrsg. von Hans-Werner Küthen (Beethoven Werke, Abteilung II, Bd. 1), München 1974, S. 124-219, Kritischer Bericht, München 1992, S. 45-57; Ders., Symphonien IV, hrsg. von Ernst Herttrich, Koreferat Christin Heitmann (Beethoven Werke, Abteilung I, Bd. 4), in Vorbereitung; Ders., Klavierauszüge, hrsg. von Ullrich Scheideler, Koreferat N. N. (Beethoven Werke, Abteilung VII, Bd. 8), in Vorbereitung. Hinzu kommt noch die Panharmonikon-Fassung von Wellingtons Sieg in: Ders., Werke für Militärmusik von Panharmonikon, hrsg. von Anja Mühlenweg unter Mitarbeit von Bernhard R. Appel nach Vorarbeiten von Heide Volckmar-Waschk, Koreferat Jens Dufner (Beethoven Werke, Abteilung II, Bd. 4), München 2017, S. 34-58, Kritischer Bericht, S. 99-102. 
b) Werk ohne Ausgabe: Die Weihe des Hauses

Carl Meisls Festspiel Die Weihe des Hauses, zu dem Beethoven die Schauspielmusik verfasste, wurde am 3. Oktober 1822 zur Wiedereröffnung des Wiener Theaters in der Josephstadt ur-

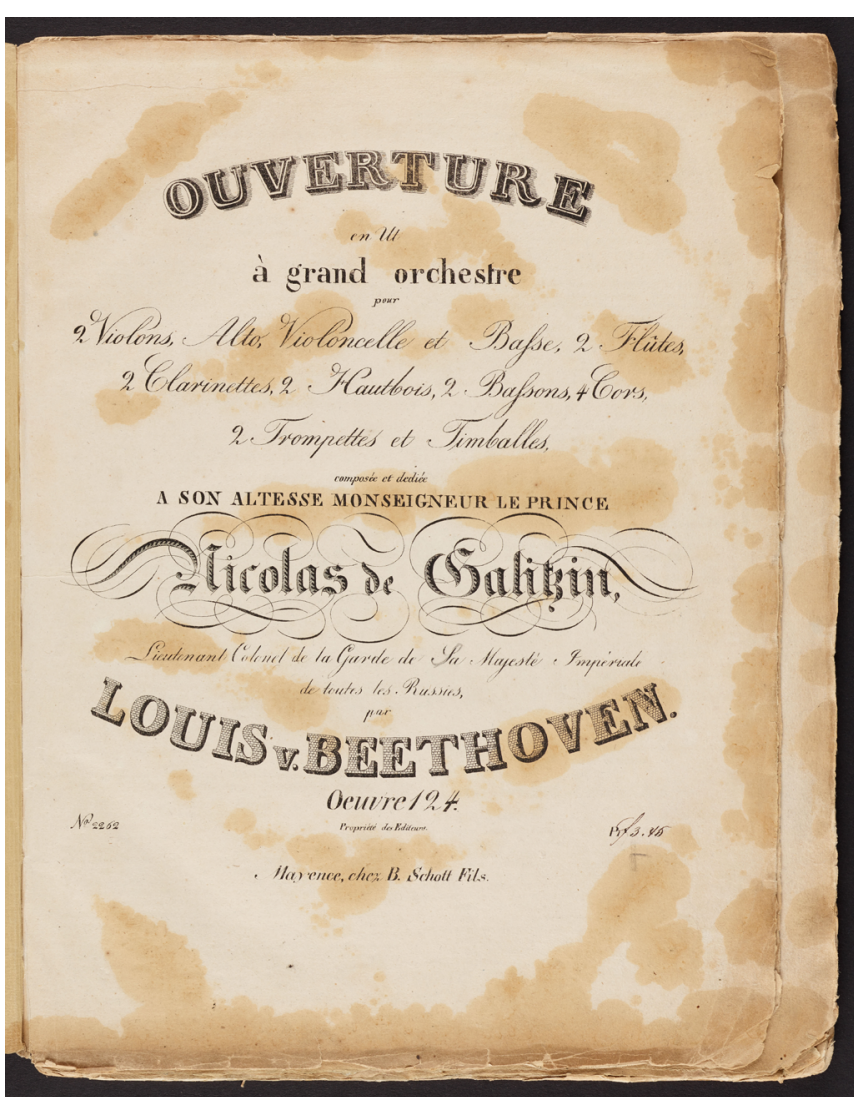

Abbildung 1: Titelseite der Partitur-Originalausgabe der Ouvertüre zu Die Weihe des Hauses op. 124, Mainz: Schott [1825] (Beethoven-Haus Bonn, C 124 / 11) aufgeführt. Beethovens Musik nimmt dabei in der wissenschaftlichen Rezeption eine Sonderstellung ein: Das Werk ist in der Beethoven-Forschung gleichsam inexistent. Die Grundlage für diese Situation hat der Komponist selbst gelegt. Er spaltete die Ouvertüre vom restlichen Notentext ab, um sie ohne Nennung des ursprünglichen Kontextes als op. 124 bei B. Schott's Söhne in Mainz zu veröffentlichen (siehe Abbildung 1 ). ${ }^{13}$ Für den Rest des Werks scheint er eine Veröffentlichung nicht in Erwägung gezogen zu haben.

Der Grund für Beethovens Entscheidung dürfte gewesen sein, dass er in zahlreichen Einzelnummern auf seine ältere Schauspielmusik zu August von Kotzebues Die Ruinen von Athen zurückgegriffen hatte. Auch deren Ouvertüre und einen "Marsch und Chor" veröffentlichte er separat als op. 113 und $114 .{ }^{14}$ Andere Nummern bot er vergeblich mehreren Verlegern an, ${ }^{15}$ so dass er nicht damit rechnen konnte, dass das Interesse der Verlage unter einem neuem Titel steigen würde. Georg Kinsky und Hans Halm übernahmen für Die Weihe

13 Dass Beethoven weder auf der Titelseite des Stimmdrucks noch auf der Titelseite der Partitur das Schauspiel erwähnt, ist umso bemerkenswerter, als er etwa auf dem Titel der Originalausgabe der Coriolan-Ouvertüre das Schauspiel und seinen Verfasser (als Widmungsträger) prominent ausstellt: "Ouverture de CoRiolan / Tragédie de Mr. de Collin / à 2 Violons, Alto, 2 Flutes / 2 Hautois, 2 Clarinettes, 2 Cors, 2 Bassons, / Trompettes, Timballes, Violoncelle et Basse / Composée et Dédieê / a Monsieur de COLLIN / Sécrétaire aulique au Service de / Sa Majesté Imp. [ériale] Roy.[ale] Ap.[ostolique] / par / LOUIS van BEETHOVEN / Op. 62 / A Vienne / Au Bureau des arts et d'industrie / A Pesth chez Schreyvogel \& Comp." (Beethoven-Haus Bonn, Sammlung H.C. Bodmer, HCB C op. 62 $=\mathrm{HCB} C \mathrm{Md}$ 9).

14 "Marsch und Chor" überarbeitete er für die Publikation. Vgl. Ludwig van Beethoven. Thematisch-bibliographisches Werkverzeichnis (wie Anm. 9), Bd. 1, S. 734.

15 Vgl. ebd., S. 727. 
des Hauses die historische Opuszahl 124, die Beethoven der Ouvertüre verliehen hatte. ${ }^{16}$ Nachdem sie die übernommenen Nummern - in Erweiterung von Beethovens Opuszahl auf das Gesamtwerk - bereits op. 113 zugeordnet hatten, blieb für den einzigen von Beethoven nachkomponierten Chorsatz eine WoO-Nummer (WoO 98). ${ }^{17}$ Obwohl selbstverständlich auch für Die Weihe des Hauses eine Übertragung der Opuszahl auf das Gesamtwerk möglich gewesen wäre, findet es im Werkverzeichnis keinen Platz.

An dieser Entscheidung, die ins neue Werkverzeichnis übernommen wurde, ${ }^{18}$ orientiert sich auch die Edition in der Neuen Beethoven-Gesamtausgabe. Der Herausgeber der Festspiele begründet die von inm gewählte Darstellung damit, dass sich eine doppelte Edition der übernommenen Nummern aus den Ruinen von Athen „eigentlich schon aus ökonomischen Gesichtspunkten wegen der prinzipiellen musikalischen Identität der Nummern in den Aufführungen von 1812 bzw. 1822 verbietet". ${ }^{19}$ Hans-Werner Küthen kündigte die von der übrigen Schauspielmusik separierte Edition der Ouvertüre im Rahmen des Bands Ouverturen und Wellingtons Sieg sogar fälschlich als "nicht im Rahmen einer Schauspiel-, Festspiel- oder Balletmusik komponiert" an. ${ }^{20}$ Wer sich für die originale Werkgestalt der Weihe des Hauses interessiert, ist daher gezwungen, diese mühevoll zu rekonstruieren (siehe Tabelle 1).

\section{c) Ein Werkkomplex: Leonore/Fidelio}

Im Bereich des Musiktheaters wird die Frage der Veränderlichkeit von Werken schon lange diskutiert. Dieser Situation trägt die Gesamtausgebe Rechnung, und so sind für Beethovens einzige Oper Leonore/Fidelio insgesamt 6 Bände geplant: Als erster Band sind die Ouvertüren zu Leonore erschienen, ${ }^{21}$ in Band 2 und 3 soll die Fassung von 1806 publiziert werden, in Band 4 Frühfassungen von Einzelnummern aus dem Jahr 1805 und in Band 5 und 6 die Fidelio-Fassung von 1814. Doch wie schon die geplante Edition von Einzelnummern von 1805 andeutet, erweist sich auch die Definition der Fassungen von 1806 und 1814 als pragmatisches Konstrukt. Denn bei der ersten Aufführung von 1814 war die überarbeitete Arie der Leonore noch nicht fertig, und auch die neu komponierte Ouvertüre lag noch nicht vor. Statt auf eine der drei LeonoreOuvertüren von 1805, 1806 und 1807 zurückzugreifen, ließ Beethoven die Ouvertüre zu den

16 Georg Kinsky, Das Werk Beethovens. Thematisch-bibliographisches Verzeichnis seiner sämtlichen vollendeten Kompositionen, nach dem Tode des Verfassers abgeschlossen und hrsg. von Hans Halm, München und Duisburg 1955, S. 366-371.

17 Ebd., S. $556 f$.

18 Vgl. Ludwig van Beethoven. Thematisch-bibliographisches Werkverzeichnis (wie Anm. 9), Bd. 1, S. 803-813, Bd. 2, S. $245 f$.

19 Helmut Hell, "Vorbemerkung" zur Edition von Die Weihe des Hauses, in: Ludwig van Beethoven, Festspiele von 1812 und 1822, hrsg. von Hellmut Hell (Beethoven Werke, Abteilung IX, Bd. 8), S. 285.

20 Hans-Werner Küthen, „Zum vorliegenden Band”, in: Beethoven, Ouverturen und Wellingtons Sieg (wie Anm. 12), S. VI-VIII, hier S. VI.

21 Ludwig van Beethoven, Ouvertüren zur Oper Leonore, hrsg. von Helga Lühning, Koreferat Christine Siegert (Beethoven Werke, Abteilung IX, Bd. 1), München 2017. 
Ruinen von Athen spielen ${ }^{22}$ - eine Möglichkeit, die in der analogen Edition nur verbal angedeutet werden kann.

\begin{tabular}{|l|l|}
\hline \multicolumn{2}{|c|}{ Tabelle 1: Die Weihe des Hauses } \\
\hline Chronologie der Nummern & Fundort in der Beethoven-Gesamtausgabe \\
\hline Ouvertüre & $\begin{array}{l}\text { NGA II/1: Ouverturen und Wellingtons Sieg, hrsg. von } \\
\text { Hans-Werner Küthen, München 1974, S. 69-123 }\end{array}$ \\
\hline Nr. 1 Chor (neu textiert) & $\begin{array}{l}\text { NGA IX/8: Festspiele von 1812 und 1822, hrsg. von Helmut } \\
\text { Hell, München 2014, Die Weihe des Hauses, S. 286-291 }\end{array}$ \\
\hline Zwischentext & $\begin{array}{l}\text { NGA IX/8, Die Weihe des Hauses, Kritischer Bericht, S. 415- } \\
\text { 417 }\end{array}$ \\
\hline Nr. 2 Duett & NGA IX/8, Die Ruinen von Athen, S. 150-156 \\
\hline Nr. 323 Marcia alla turca & NGA IX/8, Die Ruinen von Athen, S. 169-178 \\
\hline Zwischentext & NGA IX/8, Die Weihe des Hauses, Kritischer Bericht, S. 417 \\
\hline Nr. 424 Tanz mit Chor & NGA IX/8, Die Weihe des Hauses, S. 292-328 \\
\hline Zwischentext & NGA IX/8, Die Weihe des Hauses, Kritischer Bericht, S. 417f. \\
\hline Nr. 5 Harmonie auf dem Theater & $\begin{array}{l}\text { NGA IX/8, Musik: Die Ruinen von Athen, S. 179f.; Text: Die } \\
\text { Weihe des Hauses, Kritischer Bericht, S. 41825 }\end{array}$ \\
\hline Nr. 6 Marsch mit Chor & $\begin{array}{l}\text { NGA IX/8, Musik: Die Ruinen von Athen, S. 181-201; Text: } \\
\text { Die Weihe des Hauses, Kritischer Bericht, S. 418f. }\end{array}$ \\
\hline Nr. 7 Rezitativ & $\begin{array}{l}\text { NGA IX/8, Die Ruinen von Athen, S. 202-205, mit Hinwei- } \\
\text { sen zu Textänderungen in Die Weihe des Hauses, S. 329 }\end{array}$ \\
\hline Nr. 8 Chor & NGA IX/8, Die Ruinen von Athen, S. 206-219 \\
\hline Nr. 9 Arie mit Chor & $\begin{array}{l}\text { NGA IX/8, Die Ruinen von Athen, S. 220-235, mit Hinwei- } \\
\text { sen zu möglichen Textänderungen in Die Weihe des Hau- } \\
\text { ses, S. 329 }\end{array}$ \\
\hline Nr. 10 Chor & $\begin{array}{l}\text { NGA IX/8, Die Ruinen von Athen, S. 236-261, mit Hinwei- } \\
\text { sen zu Textänderungen in Die Weihe des Hauses, S. 329 }\end{array}$ \\
\hline
\end{tabular}

d) Mehrfache Autorisierung: die Neunte Symphonie op. 125

Die jüngst in der Gesamtausgabe erschienene gedruckte Edition der Neunten Symphonie berücksichtigt zahlreiche Quellen: Das Autograph ist in mehreren Einzelteilen überliefert und nicht ganz vollständig. ${ }^{26}$ Aber auch wenn es vollständig wäre, wäre es als alleinige Grundlage

22 Vgl. ebd., S. XII, 136, 173 und 186.

23 In den Ruinen von Athen Nr. 4.

24 Nicht in den Ruinen von Athen.

25 Hinzu kommen Hinweise zur Textunterlegung in Nr. 5 und 6 auf S. 329.

26 Der bei weitem größte Teil befindet sich in der Staatsbibliothek Berlin - Preußischer Kulturbesitz (Mus. ms. autogr. Beethoven, L.v. 2 und Mus. ms. autogr. Beethoven, L.v. Art. 204 [1], [2], [3a], [3b], [4] und [6]), die Coda des zweiten Satzes im Beethoven-Haus Bonn (Sammlung H.C. Bodmer, HCB Mh 2 und HCB BMh 5/45) sowie 3 Blätter 
für eine Aufführung oder für die Herstellung einer Druckausgabe untauglich - nicht nur, weil Beethoven nicht immer ordentlich schreibt, sondern auch, weil es sich um ein Arbeitsmanuskript (und nicht etwa um eine Reinschrift) handelt, das Teil eines darüber hinausgehenden Arbeitsprozesses war. Allein sechs Abschriften sah Beethoven zu unterschiedlichen Zeitpunkten durch, korrigierte und ergänzte sie und autorisierte sie für einen jeweils konkreten Zweck: Chronologisch an erster Stelle steht die Dirigierpartitur der Uraufführung, die Beethoven später zur Stichvorlage für den Schott-Verlag in Mainz umarbeiten ließ - neben dem Autograph die zweite Hauptquelle in der Neuen Gesamtausgabe. ${ }^{27}$ Leider nur fragmentarisch überliefert ist das Uraufführungs-Stimmenmaterial, das zahlreiche aufführungspraktische Eintragungen enthält. ${ }^{28}$ Dazu gehört auch eine so genannte Chordirektor-Partitur, bestehend aus Singstimmen und Instrumentalbass, die für die Einstudierung des Chores im IV. Satz verwendet wurde. ${ }^{29}$ Eine weitere Abschrift erhielt die Philharmonic Society in London, die das Werk 1817 in Auftrag gegeben hatte. ${ }^{30}$ In London wurden u.a. eine englische und eine italienische Übersetzung des Schiller'schen Textes ergänzt. An diesem Auftrag war Beethovens Schüler Ferdinand Ries, der längere Zeit in der englischen Hauptstadt lebte, maßgeblich beteiligt. Nach seiner Rückkehr ins Rheinland führte er das Werk 1825 beim Niederrheinischen Musikfest in Aachen auf. Dafür schickte Beethoven ihm eine Abschrift der ersten drei Sätze, die dann vor Ort um den, aus mitgeschickten (Uraufführungs-)Stimmen spartierten Finalsatz ergänzt wurde. ${ }^{31}$ Bei der Aufführung wurde u.a. das Eingangsrezitativ des Bariton „O Freunde, nicht diese Töne" modifiziert, offenbar um vom Tenor gesungen zu werden. ${ }^{32}$ Last but not least erhielt der Widmungsträger, der preußische König Friedrich Wilhelm III., eine von Beethoven durchgesehene Partitur. ${ }^{33}$

Die Autorisierung aller genannten Abschriften zeigt, dass man aus diesem Material sehr verschiedene, aber sämtlich autorisierte Werkfassungen rekonstruieren könnte. Die jüngst in der Gesamtausgabe erschienene gedruckte Edition, die diesen Quellenreichtum gemäß dem Druckmedium auf eine einzige Perspektive reduzieren muss, die Fassung letzter Hand, ist demgegenüber zwangsläufig recht eindimensional. Nur verzeichnet werden zudem, wie in der Neuen Gesamtausgabe üblich, Skizzen und Entwürfe, ohne diese für die Edition fruchtbar zu machen. ${ }^{34}$

zum IV. Satz in der Sammlung Malherbe des Pariser Conservatoire (F-Pn, MS-43); zwei weitere kurze Abschnitte aus dem IV. Satz sind verschollen. Vgl. Ludwig van Beethoven, Symphonie Nr. 9 d-Moll Opus 125 mit Schluß-Chor über Schillers Ode "An die Freude" für großes Orchester, 4 Solo- und 4 Chor-Stimmen, hrsg. von Beate Angelika Kraus unter Mitarbeit von Bernhard R. Appel, Koreferat Christine Siegert (Beethoven Werke, Abteilung I, Bd. 5), München 2020, Kritischer Bericht, S. 247-253.

27 US-NYj, The Juilliard Manuscript Collection, 31 B393sy no.9 1826 v.1-3; vgl. ebd., S. 255-257.

28 A-Wgm, XIII 8440/1; vgl. ebd., S. 260-262.

29 D-AAsa, HS 1084; vgl. ebd., S. 264.

30 GB-Lbl, RPS MS 5; vgl. ebd., S. $262 f$.

31 D-AAsa, HS 1098 und 1099; vgl. ebd., S. $263 f$.

32 Der neue Spitzenton ist $a^{1}$; vgl. ebd., S. 366.

33 D-B, Mus. ms. autogr. Beethoven, L.v. 30; vgl. ebd., S. $265 f$.

34 Vgl. ebd., S. $246 f$. 


\section{Zum Potential einer Digitalen Beethoven-Edition}

a) Die Fassungen der Neunten Symphonie op. 125

Das, was im analogen Medium problematisch ist, lässt sich in der digitalen Darstellung sinnvoll realisieren. Zusätzlich zu Beethovens Fassung letzter Hand ließe sich von der Neunten Symphonie die Fassung der Uraufführung, die Fassung der Londoner Erstaufführung, bei der das Chorfinale in italienischer Sprache gesungen wurde, sowie die nicht aufgeführte Fassung mit englischem Text oder die beim Niederrheinischen Musikfest in Aachen aufgeführte Fassung realisieren. Da Beethoven für alle diese ganz unterschiedlichen Aufführungen von ihm autorisiertes Notenmaterial zur Verfügung gestellt hat, liegen sie alle in seinem Werkhorizont. Dies gilt auch für die in London angefertigten Übersetzungen, denn Beethoven war bewusst, dass man dort Vokalwerke entweder auf Englisch oder Italienisch realisierte. ${ }^{35}$ Von allen Aufführungen sind weiterführende Hinweise auf die Besetzung, zumindest auf die Besetzungsstärke erhalten ${ }^{36}$ der Bericht über die Aufführung in Aachen beschreibt zudem Kürzungen, ${ }^{37}$ so dass man den Notentext mit Dokumenten weiter anreichern könnte.

Dass mit den von Beethoven unterstützten Aufführungen die Werkgeschichte der Neunten Symphonie nicht zu Ende ist, muss nicht eigens betont werden. Insofern wird das Werk umso vielgestaltiger, wenn man noch weitere Fassungen hinzuzieht, die zwar nicht mit der Person Beethoven in Beziehung gesetzt werden können, aber natürlich ihren je eigenen Beitrag zur Werküberlieferung leisten, etwa jene Gustav Mahlers, der die Blechblasinstrumente signifikant verstärkte, ${ }^{38}$ oder jene von Leonard Bernstein, der bekanntermaßen anlässlich des Falls der Berliner Mauer 1989 „Freude" durch „Freiheit" ersetzte. ${ }^{39}$

Während man in einer gedruckten Ausgabe alle diese Werkfassungen separat edieren und damit einen ausgesprochen großen Aufwand betreiben müsste, ist dies bei einer digitalen Edition nicht der Fall. Nachdem man eine Fassung ediert hat, wären nur noch die Abweichungen von anderen Fassungen hinzuzufügen. Außerdem würden in einer Druckausgabe die verschiedenen Editionen unverbunden nebeneinander stehen. Das eigentlich Bemerkenswerte, die Unterschiede zwischen den Fassungen, würden in der erdrückenden Menge von Übereinstimmendem untergehen. Zumindest wäre das Erfassen von Unterschieden ausgesprochen

35 Dass Beethoven sich des Problems bewusst war, zeigt eine Bemerkung seines Neffen Karl im Konversationsheft: „Doch ist Schiller schon ganz ins Englische übersetzt; und | es würde nicht schwer seyn, sich die Übersetzung dieses Chors zu verschaffen" (Ludwig van Beethovens Konversationshefte, Bd. 5, S. 175). Vgl. Beethoven, Symphonie Nr. 9 d-Moll (wie Anm. 26), S. 328.

36 Vgl. ebd., S. $260 f$ und 277 (Uraufführung), S. 283f. (englische Erstaufführung) und S. 286 (Aufführung beim Niederrheinischen Musikfest in Aachen).

37 "[...] das Adagio wurde zum Theil und das Menuett und Trio ganz und gar übergangen" ("Nachrichten. Vom Niederrhein", in: Allgemeine musikalische Zeitung 27, Nr. 26 (29. Juni 1825), Sp. 444-449, hier Sp. 446).

38 A-Wn, Mus. Hs. 39313.

39 Partitur in US-NY, LB1011. Bernstein nutzte die Partitur nur zur Vorbereitung. Die bei der Aufführung erstellte Videoaufnahme zeigt, dass er auswendig dirigierte. 
mühsam. Der Nutzer bzw. die Nutzerin müsste dafür eine Art Kollation der Editionen herstellen. ${ }^{40}$ Eine Codierung mit anschließendem Rendering bietet hier neue Möglichkeiten: Je nach Erkenntnisinteresse müsste die Quellenbewertung automatisiert umgesetzt werden, so dass jeweils ein anderer Notentext angezeigt wird. Abweichungen in Text und Musik sollten dabei hervorgehoben werden können. Dass man Inhomogenitäten kommentieren oder ggf. auch korrigieren müsste, liegt auf der Hand. ${ }^{41}$

\section{b) Symphonien und ihre Bearbeitungen}

Dass zwischen verschiedenen Fassungen für denselben Besetzungstyp und Bearbeitungen für bestimmte Besetzungen keine kategorialen, sondern nur graduelle Unterschiede bestehen, zeigt der Fall von Mahlers Adaption der Neunten Symphonie mit erweiterter Bläserbesetzung. So könnte auch mit Bearbeitungen editorisch vergleichbar verfahren werden wie mit Adaptionen, etwa derjenigen Mahlers. Möglichkeiten der Codierung von Bearbeitungen werden derzeit im Rahmen des Projekts Beethoven in the House insbesondere anhand von Steiners Editionen von op. 91-93 untersucht, ${ }^{42}$ wobei der Schwerpunkt der Codierung, ausgehend von Beethovens Partiturfassung, auf signifikante Passagen gelegt wird: instrumententypische Gesten und Spielweisen wie Tremolo ${ }^{43}$ oder Pizzicato, ${ }^{44}$ instrumentatorische Besonderheiten, ${ }^{45}$ semantische Interpolationen wie Zitate, ${ }^{46}$ satztechnische Auffälligkeiten wie Fugati, die Wanderung eines Motivs durch verschiedene Stimmen oder kontrapunktische Stimmführung, ${ }^{47}$ alternierende Instrumentengruppen, ${ }^{48}$ mehrere satztechnische Ebenen, ${ }^{49}$ alternierende Sforzato-Schläge, ${ }^{50}$

40 Verschiedene Darstellungsformen, um Unterschiede auch im Druck hervorzuheben, wurden im Rahmen der Edition von Haydns Arienbearbeitungen erprobt; vgl. Joseph Haydn, Bearbeitungen von Arien und Szenen anderer Komponisten, 1. Folge, München 2014 (Joseph Haydn Werke, Reihe XXVI, Bd. 3). Allerdings handelt es sich dabei in der Regel nur um zwei Fassungen.

41 Entscheidend bei der Edition unterschiedlicher Fassungen (analog wie digital) ist es, nicht eine Fassung als Quelle für die anderen zu verwenden, weil dies zu Vermischungen führt, die die Unterschiede nivellieren.

42 Das Projekt wird vom britischen Arts and Humanities Research Council und der Deutschen Forschungsgemeinschaft (Projektnummer 429039809) gefördert und als Kooperation von University of Oxford (Oxford e-Recearch Centre und Bodleian Libraries), dem RISM Digital Center in Bern, dem Musikwissenschaftlichen Seminar Detmold/Paderborn und dem Beethoven-Haus Bonn durchgeführt; Leitung: Kevin Page, Andrew Hankinson, Johannes Kepper und Christine Siegert. Es sind eine qualitative und eine quantitative Studie geplant.

43 Z. B. Wellingtons Sieg, T. 106ff. und 654ff., Siebte Symphonie, I. Satz, T. 278ff., IV. Satz, T. 32ff., 62ff., 104ff., 301ff., $427 \mathrm{ff}$., Achte Symphonie, I. Satz, T. 112ff., 190ff. und 323ff.

44 Z. B. Wellingtons Sieg, T. 423ff., Siebte Symphonie, I. Satz, T. 300ff., III. Satz, T. 65ff., IV. Satz, T. 64ff., Achte Symphonie, I. Satz, T. $235 \mathrm{ff}$.

45 Z. B. Wellingtons Sieg, T. 1ff. (Schlagwerk), T. 74ff. (Kanonenschüsse), T. 77ff. (Ratsche), T. 200ff. und 363ff. (Trommelschläge und -wirbel), Siebte Symphonie, III. Satz, T. 467ff., und IV. Satz, T. 307ff., Achte Symphonie, I. Satz, T. 112f. (Paukenwirbel).

46 Z. B. Wellingtons Sieg, T. 1ff., 31ff.

47 Z. B. Wellingtons Sieg, T. 516ff., und Siebte Symphonie, I. Satz, T. $1 \mathrm{ff}$.

48 Z. B. Siebte Symphonie, I. Satz, T. 217ff., IV. Satz, T. 1ff., und Achte Symphonie, I. Satz, T. 198ff., und IV. Satz, T. $43 \mathrm{ff}$.

49 Z. B. Siebte Symphonie, III. Satz, T. 149ff.

50 Z. B. Siebte Symphonie, IV. Satz, T. 146ff., und Achte Symphonie, III. Satz, T. 36ff. 
Raumwirkungen ${ }^{51}$ usw. $^{52}$ Erste analytische Anzeigemodalitäten hat das Projekt Beethovens Werkstatt in seinem zweiten Modul Beethoven als Bearbeiter eigener Werke entwickelt, ${ }^{53}$ die in Beethoven in the House weiter ausgebaut und differenziert werden sollen.

\section{c) Werkübergreifende Edition: Die Ruinen von Athen und Die Weihe des Hauses}

Dass die Codierung identischer Teile nur einmal vorgenommen werden muss, gilt selbstverständlich auch für den Fall von Die Ruinen von Athen und Die Weihe des Hauses, die dadurch konzeptionell als zusammengehörig dargestellt werden können. Abweichungen wären dann in einem zweiten Schritt zu ergänzen. Als Ergebnis sollte eine Anzeige beider Werke einzeln sowie eine synoptische Darstellung möglich sein. Durch die Möglichkeit der Hervorhebung von Unterschieden wird auch die Bearbeitung der einzigen in der NGA doppelt abgedruckten Nummer, des Chors Nr. 1, deutlich. Darüber hinaus wären nach dem Vorbild der Neunten Symphonie werkinterne Varianten einzubeziehen, also die als op. 114 publizierte Neufassung von "Marsch und Chor".

Die entscheidende Erweiterung gegenüber der Edition verschiedener Werkfassungen besteht in dem werkübergreifenden Konzept, das sich im Druck nicht überzeugend realisieren lässt, hingegen als entscheidende Stärke einer digitalen Edition gelten kann.

\section{d) Werkverknüpfungen: Fidelio}

Die von Beethoven selbst eröffnete Möglichkeit, die Ouvertüre der Ruinen von Athen zu Beginn einer Fidelio-Produktion zu spielen, wäre in die digitale Edition ebenfalls zu integrieren, so dass insgesamt fünf authentische Ouvertüren-Lösungen für den Leonore/Fidelio-Komplex zur Verfügung stünden. Hier bietet sich eine Verknüpfung zwischen den Werken an, denn das Aufführungsmaterial, das im Detail Aufschluss über den gespielten Notentext geben könnte, ist offenbar nicht erhalten. Die Beziehungen zwischen den verschiedenen Werken innerhalb von Beethovens Schaffen würden auf diese Weise unmittelbar erkennbar. Gleichzeitig würden Die Ruinen von Athen - und damit auch Die Weihe des Hauses - Bestandteil eines gattungsumspannenden Musiktheater-Netzwerks, wie ich es an anderer Stelle beschrieben habe. ${ }^{54}$ In

\section{Z. B. Wellingtons Sieg, 1. Abteilung: Schlacht.}

52 Die Auswahl der letztlich mit größtmöglicher Tiefe zu codierenden Passagen ist noch nicht abgeschlossen und kann von den hier genannten abweichen. Für den Hinweis auf die genannten und weitere Stellen in der VIII. Symphonie möchte ich Christin Heitmann sehr herzlich danken.

53 Vgl. Richard Sänger, "Change of Perspective - Digital Tools for the Investigation of Beethoven's Arrangements of His Own Works / Perspektivwechsel - Digitale Werkzeuge zur Untersuchung von Beethovens Eigenbearbeitungen", in: Inside Beethoven! The Audience Goes on Stage / Das begehbare Ensemble. Begleitpublikation zur Klanginstallation der Hochschule für Musik Detmold zum Septett op. 20 und Trio op. 38 (mit CD), hrsg. von Axel Berndt und Joachim Veit (Begleitpublikationen zu Ausstellungen des Beethoven-Hauses, Bd. 28), Bonn 2019, S. 80-97.

54 Christine Siegert und Kristin Herold, "Die Gattung als vernetzte Struktur. Überlegungen zur Oper um 1800", in: „Ei, dem alten Herrn, zoll' ich Achtung gern'". Festschrift für Joachim Veit zum 60. Geburtstag, hrsg. von Kristina Richts und Peter Stadler für den Virtuellen Forschungsverbund Edirom, München 2016, S. 671-702. Ebd., S. 682702, sind insgesamt 154 Werke verzeichnet, die über Einlagen oder die Verwendung derselben Nummern mit 
Bezug auf Fidelio konnte ich dort Übernahmen der Ouvertüre in Karl Ludwig Unraths Pasticcio Musikalischer Wettstreit, ${ }^{55}$ der langsamen Einleitung zu Florestans Rezitativ "Gott, welch' Dunkel hier" in Bernhard Breuers Pasticcio Die Kölner in Paris ${ }^{56}$ sowie des Schlussgesangs "Wer ein holdes Weib errungen" in Adolf Müllers Posse Einen Jux will er sich machen verzeichnen. ${ }^{57}$ Für alle drei Stücke sind im Répertoire International des Sources Musicales Quellen nachgewiesen, ${ }^{58}$ so dass die jeweiligen Einzelnummern unter der Maßgabe des skizzierten erweiterten Werkbegriffs selbstverständlich in eine Digitale Beethoven-Edition zu integrieren wären. Doch darüber hinaus sollten die "Zielopern", in die Beethovens Musik einging, auch als ganze in eine Digitale Beethoven-Edition einbezogen werden, da die Nummern nur so adäquat in ihrem Zusammenhang präsentiert werden können und nur so ein umfassendes Verständnis von Beethovens Schaffen, wie es sich den Zeitgenossen darstellte, möglich ist. Dass es sich dabei um Werke ganz unterschiedlicher Stilhöhe handelt, ist sicherlich von besonderem Reiz. Der Sachverhalt, dass in den drei genannten Werken Nummern ganz unterschiedlicher Komponisten zusammengeführt wurden, macht eine derartig konzipierte Digitale Beethoven-Edition anschlussfähig für andere Projekte (s. Tabelle 2).

e) Verbindungen über Metatexte und Materialität

Auch zwischen der Ouvertüre zu Die Weihe des Hauses und der Neunten Symphonie ergibt sich eine Verbindung: Die beiden Werke erschienen, ebenso wie das Opferlied op. 121b, ${ }^{59}$ das Bundeslied op. 122, ${ }^{60}$ die Missa solemnis op. 123, die Bagatellen op. 126, das Es-Dur-Streichquartett op. 127 und Der Kuß op. 128 in den Jahren 1825 bis 1827 im Schott-Verlag.

Die Ouvertüre kam im Dezember 1825 heraus. In der Ausgabe waren, hintereinander auf einer Seite, drei Verlagstexte abgedruckt: Zunächst eine gemeinsame Subskriptionsanzeige für die Missa solemnis, die Ouvertüre und die Symphonie, im Anschluss eine weitere Verlagsankündigung für vier Fassungen des Streichquartetts op. 127 (als Partitur- und Stimmenausgabe sowie in Bearbeitungen für Klavier zu zwei und zu vier Händen) und an dritter Stelle das Verlagsprivileg für alle genannten Ausgaben. In späteren Auflagen sollen diese Anzeigen, Kinsky/

anderen Musiktheaterwerken in Beziehung stehen. Durch die beiden Schauspielmusiken Beethovens wächst das Netzwerk auf 156 Werke an.

55 Ebd., S. 677.

56 Ebd., S. 679.

57 Ebd., S. 679.

$58<$ https://opac.rism.info/index.php?id=4> (15.10.2020).

59 Sowohl das bei Schott erschienene Opferlied als auch die bei Steiner und Comp. erschienenen Variationen über Wenzel Müllers „Ich bin der Schneider Kakadu” erhielten die Opuszahl 121, so dass von der Nachwelt die Variationen als op. 121a vom Opferlied op. 121b unterschieden werden (wohl erstmals 1851; vgl. Ludwig van Beethoven. Thematisch-bibliographisches Werkverzeichnis [wie Anm. 9], Bd. 1, S. 780). Vom Opferlied erschienen drei Originalausgaben: als Partitur, Stimmen und Klavierauszug. Außerdem kamen in anderen Verlagen Bearbeitungen für Gesang und Klavier bzw. Harfe heraus (vgl. ebd., S. 782f.).

60 Vom Bundeslied erschienen mit Partitur, Stimmen und Klavierauszug ebenfalls drei Originalausgaben (vgl. ebd., S. 786f.). 


\begin{tabular}{|c|c|c|}
\hline \multicolumn{3}{|c|}{ Tabelle 2: Musiktheater unter Einbeziehung von Fidelio } \\
\hline $\begin{array}{l}\text { Karl Ludwig Unrath, Ein musi- } \\
\text { kalischer Wettstreit }\end{array}$ & $\begin{array}{l}\text { Bernhard Breuer, Die Kölner in } \\
\text { Paris }\end{array}$ & $\begin{array}{l}\text { Adolf Müller, Einen Jux will er } \\
\text { sich machen }\end{array}$ \\
\hline $\begin{array}{l}\text { Wolfgang Amadé Mozart, La } \\
\text { clemenza di Tito, Sinfonia }\end{array}$ & $\begin{array}{l}\text { Drechsler, Der Bauer als Millio- } \\
\text { när, "So mancher steht herum" }\end{array}$ & $\begin{array}{l}\text { Mozart, Die Entführung aus dem } \\
\text { Serail, "Nie werd' ich deine Huld } \\
\text { verkennen“ }\end{array}$ \\
\hline Beethoven, Fidelio, Ouvertüre & $\begin{array}{l}\text { Beethoven, Fidelio, "Gott, welch' } \\
\text { Dunkel hier" (langsame Einlei- } \\
\text { tung) }\end{array}$ & $\begin{array}{l}\text { Weber, Euryanthe, „O Seligkeit, } \\
\text { dich fass ich kaum" }\end{array}$ \\
\hline $\begin{array}{l}\text { Gioacchino Rossini, Guillaume } \\
\text { Tell, Ouvertüre }\end{array}$ & $\begin{array}{l}\text { Carl Maria von Weber, Der Frei- } \\
\text { schütz, "Wir winden dir den } \\
\text { Jungfernkranz" }\end{array}$ & $\begin{array}{l}\text { Beethoven, Fidelio, "Wer ein } \\
\text { holdes Weib errungen“ }\end{array}$ \\
\hline Wallerstein, Jahrmarkts-Polka & $\begin{array}{l}\text { Daniel-François-Esprit Auber, La } \\
\text { muette de Portici, "Au marché } \\
\text { qui vient de s'ouvrir" }\end{array}$ & $\begin{array}{l}\text { Donizetti, Lucrezia Borgia, „Lass } \\
\text { den heutigen Tag uns genießen }\end{array}$ \\
\hline $\begin{array}{l}\text { Gaetano Donizetti, Lucrezia } \\
\text { Borgia, Duett }\end{array}$ & $\begin{array}{l}\text { Pierre Gaveaux, Le petit matelot, } \\
\text { "Contre les chagrins de la vie" }\end{array}$ & Ferdinand Hérold, Zampa \\
\hline Mozart, Le nozze di Figaro, Arie & $\begin{array}{l}\text { Rossini, Tancredi, „Di tanti pal- } \\
\text { piti” }\end{array}$ & \\
\hline \multicolumn{3}{|l|}{ Rossini, Otello, Arie } \\
\hline \multicolumn{3}{|l|}{$\begin{array}{l}\text { Giacomo Meyerbeer, Robert le } \\
\text { Diable, Szene }\end{array}$} \\
\hline \multicolumn{3}{|l|}{ Balfe, Die vier Haimonskinder } \\
\hline Johann Strauß, Walzer & & \\
\hline
\end{tabular}

Halm zufolge, durch das gemeinsame Subskribentenverzeichnis für op. 123-125 ersetzt worden sein. ${ }^{61}$ In jedem Fall ist es in den frühen Partitur-Ausgaben der Neunten Symphonie und der Missa solemnis enthalten. Bei der Codierung sind derartige Metatexte selbstverständlich einzubeziehen, so dass sich auf dieser Ebene weitere Verknüpfungsmöglichkeiten ergeben. Für das Konzept einer Digitalen Beethoven-Edition sind die Werke also sowohl auf der Ebene der Quellen als auch auf der Ebene der Metatexte von Bedeutung.

Hinzu kommt der Befund, dass einzelne Quellen verschiedene Werke enthalten können, was im Fall von Skizzen und Entwürfen eher die Regel als die Ausnahme darstellt. Skizzenbücher und -blätter sollten also auf eine Weise codiert werden, die sowohl die Heterogenität ihres Inhalts in der konkreten räumlichen Disposition wiedergibt, als auch die (ggf. hypothetische) Zuordnung einzelner Skizzen zu den edierten Werken, ggf. sogar zu klar definierten Positionen im Werk ermöglicht.

61 Kinsky, Das Werk Beethovens (wie Anm. 16), S. 369. Laut Ludwig van Beethoven. Thematisch-bibliographisches Werkverzeichnis (wie Anm. 9), Bd. 1, S. 811 ist „ein solches Exemplar [...] für Op. 124 bisher nicht nachgewiesen." 


\section{Konzeptionelle Überlegungen}

a) Quellen versus Fassungen

Nicht nur die Zuordnung von Quellen zu Werken kann vielschichtig sein, sondern auch die Zuordnung von Quellen zu Fassungen funktioniert nicht so reibungslos, wie es auf den ersten Blick scheinen könnte. Im Fall der Neunten Symphonie lassen sich, wie gezeigt, die Fassung der Uraufführung, die Fassung letzter Hand, die Fassung der Londoner Erstaufführung usw. unterscheiden. Aber die Quellen repräsentieren nicht uneingeschränkt diese Fassungen; sie sind mit ihnen nicht deckungsgleich. Zur Fassung der Uraufführung gehören selbstverständlich die Uraufführungsstimmen. Aber nicht jede aufführungspraktische Bemerkung, die sie enthalten, ist der Uraufführung zuzuordnen, da die Stimmen bis ins späte 19. Jahrhundert hinein als Aufführungsmaterial verwendet wurden. Der Uraufführungs-Anteil an diesem Material ist also zunächst zu bestimmen und von späteren Anteilen abzugrenzen. Außerdem bilden die Stimmen keineswegs die einzige Quelle für die Fassung der Uraufführung. Auch die spätere Stichvorlage in ihrer Fassung als Dirigierpartitur gehört zwingend dazu. In dieser Partitur lassen sich heute drei Textschichten unterscheiden. ${ }^{62}$ Die Fassung der Uraufführung ist bereits die zweite Textschicht in dieser Quelle, die indes über weite Strecken mit der ersten Textschicht identisch ist. Die dritte Textschicht repräsentiert demgegenüber die Fassung letzter Hand. Doch damit nicht genug: Um die Fassung der Uraufführung so weit wie möglich zu rekonstruieren, wäre es zudem notwendig, die Abschriften für Ferdinand Ries und für den preußischen König heranzuziehen, die auf der Basis von Uraufführungsstimmen spartiert wurden. Beide haben zudem noch eigene Anteile. So ist die Widmungspartitur die einzige von Beethoven autorisierte Werkniederschrift, die Metronomangaben enthält. Sie wurden erst 1826, mehr als zwei Jahre nach der Uraufführung, festgelegt. Es sind also spezifische Inhalte (mehrerer) Quellen in einer je zu definierenden Kombination, die eine jeweils zu rekonstruierende Fassung des Werks wiederspiegeln.

Natürlich stellt sich die Frage, wie eine Datenstruktur für eine solchermaßen skizzierte Edition aussehen könnte. Verwendet man die Terminologie des FRBR-Modells, ${ }^{63}$ bedeutet dies, dass das Verhältnis von Expression (z. B. die Fassung) und Manifestationen (Quellen, die im Fall von Handschriften mit der Kategorie des Exemplars zusammenfallen) ein jeweils erst zu definierendes ist, das durch die editorische Arbeit im Wortsinn erarbeitet werden muss. Insbesondere kann sich die Expression als Derivat aus der Kombination mehrerer Manifestationen konstituieren, was ihren Status als gedankliches Konstrukt hervorhebt, das nur nach einer intensiven editorischen Tätigkeit überhaupt erschlossen werden kann. ${ }^{64}$ Insofern wäre bei einer derartigen

62 Vgl. Beethoven, Symphonie Nr. 9 d-Moll (wie Anm. 26), S. $255 f$.

63 <https://www.ifla.org/publications/functional-requirements-for-bibliographic-records> (11.10.2020).

64 Katrin Bicher und Barbara Wiermann, „Normdaten zu ,Werken der Musik' und ihr Potenzial für die digitale Musikwissenschaft, in: Bibliothek. Forschung und Praxis 42 (2018), S. 222-235, hier S. 223, differenzieren dabei zwischen "abstrakte[n]" Werken und "noch nicht materialisierte[n]" Expressionen. 
digitalen Edition die synthetisierende Edition einer definierten Fassung auf einer Zwischenebene zwischen der Expression, die sie konzeptionell repräsentiert, und der Manifestation, der sie selbst materialiter (analog oder digital) zugehört, anzusiedeln. Unterschiede müssten auf dieser Ebene ebenso sichtbar gemacht werden können wie im Vergleich der verschiedenen Quellencodierungen.

\section{b) Historizität der Fassungen}

Während das FRBR-Modell ermöglicht, verschiedene Fassungen eines Werks gleichberechtigt nebeneinanderzustellen, relativiert es die chronologische Abfolge der verschiedenen Fassungen, die sich jedoch in der Regel nachweisen oder zumindest durch Quellenvergleich erschlieBen lassen. Dabei ist die Chronologie mit philologisch dokumentierbaren Spezifika der einzelnen Fassungen verknüpft, die in einer traditionellen Edition üblicherweise mit Hilfe eines Stemmas visualisiert werden. Eine solche stemmatische Darstellung sollte - über die von FRBR vorgesehenen relationalen Beschreibungsmöglichkeiten hinaus - auch in einer digitalen Edition vorgesehen werden, da sie Gemeinsamkeiten und Unterschiede qualifiziert und so die Beziehungen zwischen den Quellen und Fassungen verdeutlicht. Ideal wäre zu diesem Zweck eine stemmatische Anordnung, in die man, wie bei der synoptischen Darstellung der EdiromAnzeige ${ }^{65}$ abgegrenzte Quellenausschnitte per Mausklick einbinden könnte.

\section{c) Multiperspektivische Zugangsmöglichkeiten}

Dass sich ein Werk nicht in seinen Handschriften erschöpft, brauche ich in diesem Rahmen sicherlich nicht zu betonen. Auch nicht, dass Handschriften, Drucke, Audiodateien und Weiteres ein recht heterogenes Quellenkorpus bilden, wenn man sich dem Werk in seiner umfassenden Bedeutung tatsächlich philologisch nähern möchte. Insofern stellt sich zunächst die Frage der Strukturierung - oder vielleicht auch nur Vorstrukturierung - dieses umfangreichen Materials.

Das Beethoven-Haus Bonn verfügt bereits über ein Modell dafür: Wie bewahren nicht nur die umfassendste und vielseitigste Beethoven-Sammlung weltweit, sondern haben auch zahlreiche Bestände digitalisiert und machen diese über das sogenannte Digitale Beethoven-Archiv allen Interessierten kostenlos zugänglich. ${ }^{66}$ Einer der Zugänge im Digitalen Archiv ist dabei der nach Werken.

Er ist nach Gattungen untergliedert, ganz ähnlich wie die Neue Beethoven-Gesamtausgabe. Diese Gliederung der Gesamtausgabe ist allerdings dem Sachverhalt geschuldet, dass eine gedruckte Ausgabe in Bänden erscheint und auch für die Musikpraxis nutzbar sein soll, und dass es gilt, vor diesem Hintergrund die Werke sinnvoll zusammenfassen. Für eine digitale Ausgabe gilt diese unveränderliche Zuordnung nicht. Stattdessen ist es sinnvoll, eine flexible Gliederung nach verschiedenen Kriterien vorzusehen bzw. verschiedene Zugänge zu ermöglichen, darun-

$65 \mathrm{Zu}$ den verschiedenen Edirom-Tools vgl. <https://www.edirom.de/werkzeuge.html> (15.10.2020).

$66<$ https://www.beethoven.de/de/archive/list> (11.10.2020). 
ter durchaus den nach Gattungen, aber auch, zum Beispiel, einen chronologischen in der Reihenfolge der Werkentstehung oder einen Zugang nach Opuszahlen sortiert. Natürlich könnte man sich weitere Zugänge vorstellen: Beispielsweise nach Textdichter*innen, Widmungsträger*innen oder Verleger*innen, nach Uraufführungsdaten oder Orten der Uraufführung oder der Komposition, nach Interpret*innen usw. Jedes Werk, jede Fassung und jede Quelle stünde dabei für sich und würde flexible Beziehungen zu anderen eingehen.

\section{d) Unabgeschlossenheit der Edition}

Zwar sind einer digitalen Edition theoretisch keine Grenzen gesetzt - und die vorgestellten Überlegungen lassen die Vielzahl und Vielgestaltigkeit der Möglichkeiten erahnen -, aber mit praktischen Grenzen wird jede Edition konfrontiert. Dies gilt für eine digitale Edition, die vor dem Hintergrund des hier zugrunde gelegten Werkbegriffs prinzipiell unabgeschlossen ist, umso mehr. Um dieses Problem zumindest ansatzweise in den Griff zu bekommen, wird es also notwendig sein, die der Edition zugrundeliegenden Erkenntnisinteressen genau zu definieren ${ }^{67}$ und verschiedene Grade der Tiefenerschließung zu ermöglichen. ${ }^{68}$

Neben den verschiedenen Graden der Tiefenerschließung sind auch verschiedene Typen von Tiefenerschließungen zu berücksichtigen. So ist etwa die mannigfaltige Gestalt von Druckausgaben in der Beethoven-Forschung bislang erst ansatzweise ins Bewusstsein gedrungen. Doch hat sich im Rahmen unserer analogen Gesamtausgabe herausgestellt, dass die Kategorisierung nach sogenannten Titelauflagen ${ }^{69}$ für die philologische Forschung nur ein erster Schritt sein kann. Da es uns um die Herstellung von Notentexten geht, sind Auflagen sinnvollerweise in erster Linie nach Änderungen im Notentext zu unterscheiden. Das heißt: ein veränderter Titel markiert aller Wahrscheinlichkeit nach eine neue Auflage ${ }^{70}$ aber ein gleich bleibender Titel bedeutet keineswegs, dass es sich um ein und dieselbe Auflage handelt. In diesem Zusammenhang kommt die vierte FRBR-Entität zum Tragen, das Exemplar.

Es ist tatsächlich auch unter philologischen Gesichtspunkten sinnvoll, möglichst viele Exemplare eines Drucks zu untersuchen, denn die Zahl der überlieferten Exemplare kann etwas über ihre Funktion aussagen. So hat die Untersuchung von über 50 Exemplaren der Originalausgabe von Beethovens „Diabelli-Variationen" op. 120 ergeben, dass es sich bei der ersten Auflage um eine veritable Verkaufsauflage handelt, und nicht nur um Korrekturabzüge, wie man annehmen könnte, weil Beethoven sich gegenüber seinem Adlatus Anton Schindler über "sehr viele Fehler

67 Diese Notwendigkeit besteht prinzipiell bei jeder Edition, doch scheint sie aufgrund der in der Musikwissenschaft etablierten langjährigen philologischen Tradition Herausgeber*innen von gedruckten Ausgaben möglicherweise gelegentlich weniger virulent.

68 Verschiedene Möglichkeiten der Tiefenerschließung auszuloten, ist eine zentrale Fragestellung insbesondere der quantitativen Studie von Beethoven in the House.

69 Vgl. Ludwig van Beethoven. Thematisch-bibliographisches Werkverzeichnis (wie Anm. 9), Bd. 1, S.32*f.

70 Bei der Originalausgabe der "Diabelli-Variationen” wurden parallel verschiedene Titel verwendet. 
[...] in den Variationen Bey Diabelli"71 beschwert. Aber die Zahl der überlieferten Exemplare gut ein Viertel des Gesamtbestands - ist für eine derartige Interpretation einfach zu groß, zumal sich darunter kein einziges Korrekturexemplar befindet.

Eine dritte Herausforderung ist die Kombination verschiedener Quellentypen innerhalb einer Expression. Es tragen also nicht nur verschiedene Handschriften zu einer Fassung bei, sondern eine Fassung kann auch beispielsweise durch eine Tonaufnahme und eine dazugehörige Notenausgabe repräsentiert sein. Hier sind ganz neue Formen der Konstitution von Beziehungen und der Kommentierung vonnöten, über die Andreas Münzmay kürzlich grundlegende Überlegungen vorgestellt hat. ${ }^{72}$

Im Vergleich zu traditionellen Werkausgaben ist bei dem hier skizzierten Konzept einer digitalen Ausgabe sowohl die Quellengrundlage aufgrund des inklusiven Ansatzes als auch das Erkenntnispotential durch die multiperspektivischen Zugangsweisen also deutlich erweitert. Dies am Beispiel Ludwig van Beethovens zu erproben, ist eine Aufgabe für die Zukunft.

Zitation: Christine Siegert, „Komponisten-Gesamtausgaben im digitalen Zeitalter: Perspektiven und Reflexionen am Beispiel Ludwig van Beethovens", in: Brückenschläge zwischen Musikwissenschaft und Informatik. Theoretische und praktische Aspekte der Kooperation, in Verbindung mit der Fachgruppe Digitale Musikwissenschaft hrsg. von Stefanie Acquavella-Rauch, Andreas Münzmay und Joachim Veit (= Musikwissenschaft: Aktuelle Perspektiven. Bericht über die Jahrestagung der Gesellschaft für Musikforschung 2019 in Paderborn und Detmold, Bd. 3), Detmold, Musikwissenschaftliches Seminar der Universität Paderborn und der Hochschule für Musik Detmold, 2020, S. 61-76, DOI: $10.25366 / 2020.95$

71 Beethoven an Anton Schindler, kurz nach dem 3. Juni 1823, in: Ludwig van Beethoven. Briefwechsel Gesamtausgabe (wie Anm. 11), Bd. 5: 1823-1824, München 1996, S. 149, Nr. 1670a.

72 Vgl. Andreas Münzmay und Christine Siegert, „Phonographischer Text, Interpretation und Aufführungsmaterial als kritisch edierbarer Sachzusammenhang. Ein Beitrag zur Theorie der Edition von Klangdokumenten", in: editio 33 (2019), S. 10-30, hier S. 15-30. 


\section{Abstract}

In the field of edition philology a diverse range of digital approaches is being put to the test. Taking Ludwig van Beethoven as a basis, this article demonstrates the limits of printed editions and presents preliminary considerations for a genuinely digital edition of his works. Various versions of the Ninth Symphony, the publisher Sigmund Anton Steiner's publication concept for the Seventh and Eighth Symphonies and Wellingtons Sieg, which incorporated arrangements for highly diverse scorings, as well as the use of single numbers from the opera Fidelio in other music theatre works of the time, all serve as examples. The significance of metatexts and connections in terms of materiality are also discussed. Conceptional principles of such a Digital Beethoven Edition would include an inclusive approach allowing for multiple perspectives, which greatly expands both the number of sources on which an edition is based and the potential for insight, in contrast with traditional editions.

\section{Kurzvita}

Christine Siegert leitet seit 2015 das Forschungszentrum Beethoven-Archiv am BeethovenHaus Bonn. Promotion 2003 über Luigi Cherubini. Danach Wissenschaftliche Mitarbeiterin u.a. am Joseph Haydn-Institut Köln und am Akademieprojekt "OPERA - Spektrum des europäischen Musiktheaters in Einzeleditionen" (Universität Bayreuth). 2010-2015 Juniorprofessorin an der Universität der Künste Berlin, ab 2013 Leitung des von der Einstein Stiftung Berlin finanzierten Forschungsprojekts „A Cosmopolitan Composer in Pre-Revolutionary Europe - Giuseppe Sarti". 2013-2017 Sprecherin der Kommission für Auslandsstudien der Gesellschaft für Musikforschung, seitdem im Beirat der Gesellschaft; seit August 2020 Mitglied der Wissenschaftlichen Kommission der Union der deutschen Akademien der Wissenschaften. Ab 2016 Generalherausgeberin der Beethoven-Gesamtausgabe, seit 2020 Leitung des Bonner Teils des Kooperationsprojekts "Beethoven in the House" (Kooperation mit dem Musikwissenschaftlichen Seminar Detmold-Paderborn und der Oxford University, gefördert von der DFG und dem britischen AHRC). 



\section{Brückenschläge zwischen Musikwissenschaft und Informatik}

Theoretische und praktische Aspekte der Kooperation

Herausgegeben von Stefanie Acquavella-Rauch,

Andreas Münzmay und Joachim Veit 
Brückenschläge zwischen Musikwissenschaft und Informatik 


\section{Musikwissenschaft: Aktuelle Perspektiven}

Bericht über die Jahrestagung der Gesellschaft für Musikforschung 2019 in Paderborn und Detmold

Herausgegeben von Rebecca Grotjahn und Nina Jaeschke

Band 3 


\section{Brückenschläge zwischen Musikwissenschaft und Informatik}

Theoretische und praktische Aspekte der Kooperation

Beiträge der Symposien zur Digitalen Musikwissenschaft

Osnabrück 2018 und Paderborn 2019

im Rahmen der Jahrestagungen der Gesellschaft für Musikforschung

In Verbindung mit der Fachgruppe Digitale Musikwissenschaft herausgegeben von

Stefanie Acquavella-Rauch, Andreas Münzmay und Joachim Veit

Detmold: Musikwissenschaftliches Seminar der Universität Paderborn und der Hochschule für Musik Detmold 2020 
DOI: $10.25366 / 2020.87$

Online-Version verfügbar unter der Lizenz: Urheberrecht 1.0, $<$ https://rightsstatements.org/page/InC/1.0/?language =de >

Bibliografische Information der Deutschen Nationalbibliothek

Die Deutsche Nationalbibliothek verzeichnet diese Publikation in der Deutschen Nationalbibliografie; detaillierte bibliografische Daten sind im Internet über http://dnb.d-nb.de abrufbar.

\section{Impressum}

Redaktion: Stefanie Acquavella-Rauch, Andreas Münzmay und Joachim Veit Satz: Nina Jaeschke und Joachim Veit

(C) Musikwissenschaftliches Seminar der Universität Paderborn und der Hochschule für Musik Detmold 2020 


\section{INHALT}

Rebecca Grotjahn, Nina Jaeschke

Vorwort zu Band 1-3

IX

Stefanie Acquavella-Rauch, Andreas Münzmay, Joachim Veit

Brückenschläge zwischen Musikwissenschaft und Informatik - Vorbemerkung

$\mathrm{XI}$

\section{KOLLABORATIONEN - KO-LABORATORIEN}

\section{Reinhard Keil}

Der Computer als Denkzeug für hermeneutische Arbeit

\section{Ulrich Konrad}

Philologie und Digitalität. Perspektiven für die Musikwissenschaft im Kontext fächerübergreifender Institutionen

\section{Gudrun Oevel}

Infrastruktureinrichtungen in Forschungsprojekten - Spagat oder Chance?

\section{Dennis Ried}

Erhebung, Transformation und Präsentation digitaler Forschungsdaten

Anna Neovesky, Frederic von Vlahovits

IncipitSearch - Leitfaden zur Zusammenarbeit

Elisabeth Treydte

Clara Schumann \#digital. 40 Jahre Archiv Frau und Musik und der Start in die Digitalisierung

\section{TEXT/DATEN/PROZESSE}

\section{Christine Siegert}

Komponisten-Gesamtausgaben im digitalen Zeitalter: Perspektiven

und Reflexionen am Beispiel Ludwig van Beethovens

Markus Neuwirth, Johannes Hentschel, Martin Rohrmeier

Perspectives of Musical Corpus Studies: The Annotated Mozart Sonatas

Agnes Amminger, Franz Kelnreiter

Leopold Mozarts „Gründliche Violinschule". Zur Textcodierung und -präsentation einer digitalen Edition

\section{Oleksii Sapov}

Algorithmische Automatisierung komplexer Notationsregeln in MEI-XML am Beispiel von Versetzungszeichen 


\section{Susanne Cox, Richard Sänger}

Digitale Fassungsvergleiche am Beispiel von Beethovens Eigenbearbeitungen

Agnes Seipelt

Digitale Edition und Harmonische Analyse mit MEI von Anton Bruckners

Studienbuch

Stefanie Acquavella-Rauch

Musikalische Schaffensprozesse 2.0 - Inkorporation audiovisueller Medien

der populären Musik in Methoden der digitalen Edition

\section{DIGITAL(ISIERT)E MATERIALITÄTEN}

\section{Miriam Akkermann}

(Musik)Instrument (im) Computer

\section{Daniel Fütterer}

Herausforderungen bei der Kodierung von Paratext am Beispiel Neuer Musik mit Live-Elektronik

\section{Matthias Pasdzierny}

How much is the glitch? Das digitale Paradigma als Herausforderung

und Chance für die historische Musikwissenschaft

\section{Shintaro Miyazaki}

Musik für Maschinen?! - Wo sich die Wissenschaft der Medien, des Computers und der Musik treffen und wie sie zusammenarbeiten könnten

\section{MUSIKGESCHICHTE(N) IM NETZ}

\section{Matthias Tischer}

Musikgeschichte der DDR: Ein Pilotprojekt zur digitalen Musikvermittlung

Annette van Dyck-Hemming, Jan Eberhardt, Melanie Wald-Fuhrmann

Ansätze zur Analyse historischer Netzwerke mit Neo4j® - Aus der Projekt-Werkstatt der Datenbank zur Fachgeschichte der Musikwissenschaft

Axel Beer, Martin Bierwisch, Kristina Krämer

Das MMM2 - Ein regionalgeschichtliches Onlinelexikon der Arbeitsgemeinschaft für mittelrheinische Musikgeschichte

\section{Matej Santi}

Was erzählt Fritz Kreislers Geige?

\section{Elias Berner}

Alle Menschen werden Brüder?! Ein historisches Dokument aus dem

Nationalsozialismus in den sozialen Medien 
Gabriele Buschmeier in memoriam 